学位論文

Yokukansan Reduces Cuprizone-Induced Demyeliantion in the Corpus Callosum Through Anti-inflammatory Effects on Microglia

抑肝散はミクログリアに抗炎症作用を示し， 脳梁におけるクプリゾン誘発性脱髄を抑制する

旭川医科大学大学院医学系研究科博士課程医学専攻

野村太一

（板東良雄，Hua You，田中達英，吉田成孝） 


\title{
Yokukansan Reduces Cuprizone-Induced Demyelination in the Corpus Callosum Through Anti-inflammatory Effects on Microglia
}

\author{
Taichi Nomura $^{1}$ Yoshio Bando ${ }^{1}$ Hua You ${ }^{1,2} \cdot$ Tatsuhide Tanaka $^{1,3}$. \\ Shigetaka Yoshida ${ }^{1}$
}

Received: 27 May 2017 / Revised: 17 August 2017 / Accepted: 11 September 2017 / Published online: 19 September 2017

(C) Springer Science+Business Media, LLC 2017

\begin{abstract}
Multiple sclerosis (MS) is an inflammatory demyelinating disease of the central nervous system (CNS). The release of inflammatory cytokines and pro-oxidant molecules from microglia has been shown to play a key role in the pathology of MS. Thus, suppression of microglial cell activation is an attractive therapeutic option. Yokukansan, a traditional Japanese herbal medicine, has been shown to suppress microglial activity in the CNS. However, whether or not yokukansan reduces demyelination observed in the CNS during MS remains unknown. In this study, female C57BL/6 mice were fed a diet containing $0.2 \%$ cuprizone (bis-cyclohexanone oxaldihydrazone) to induce demyelination in the corpus callosum. We investigated whether or not yokukansan reduces cuprizone-induced demyelination using immunohistochemical analyses. Furthermore, we examined the in vitro anti-inflammatory effects of yokukansan on LPSstimulated BV2 cells, a murine microglial cell line. Luxol fast blue staining and immunostaining for myelin basic protein demonstrated that yokukansan reduces demyelination of the corpora callosa of cuprizone-fed mice. In addition, yokukansan significantly decreased the number of activated microglial cells in the corpora callosa of cuprizone-fed mice. Furthermore, treatment with $500 \mu \mathrm{g} / \mathrm{ml}$ yokukansan
\end{abstract}

Taichi Nomura

t-nomura@asahikawa-med.ac.jp

1 Department of Functional Anatomy and Neuroscience, Asahikawa Medical University, Midorigaoka-higashi

2-1-1-1, Asahikawa, Hokkaido 078-8510, Japan

2 Affiliated Hospital of Academy of Military Medical Sciences, No.8 East Main Street, Fengtai District 100071, Beijing, China

3 Present Address: Department of Anatomy and Neuroscience, Faculty of Medicine, Nara Medical University, 840 Shijo-cho, Kashihara, Nara 634-8521, Japan suppressed the expression of interleukin- $1 \beta$ and inducible nitric-oxide synthase mRNA and protein in LPS-stimulated BV2 cells. These findings suggest that yokukansan reduces demyelination owing to anti-inflammatory effects on microglia. As yokukansan has few adverse effects, yokukansan has the potential to be a novel option to treat MS.

Keywords Yokukansan - Microglia - Demyelination · Multiple sclerosis $\cdot$ Cuprizone $\cdot$ BV2 cells

\section{Introduction}

Multiple sclerosis (MS) is an immune-mediated demyelinating disease of the central nervous system (CNS). In the pathology of MS, activation of innate immune cells and microglial cells, along with perivascular infiltration of $\mathrm{T}$ lymphocytes and macrophages, is typically observed [1,2]. In patients with active MS, these cells produce inflammatory cytokines, including IL- $1 \beta$ and pro-oxidant molecules such as inducible nitric oxide synthase (iNOS) at the lesion site $[3,4]$. Accumulating evidence indicates that microglial cells produce these molecules and have a key role in the initial and sustained immune responses observed in the pathology of MS [1-5]. Thus, strategies designed to suppress proinflammatory microglial activity are an attractive approach to the treatment of MS.

Yokukansan, a traditional Japanese herbal medicine, has been reported to have various ameliorative effects on the neurological symptoms of patients with Alzheimer's disease or schizophrenia [6-10]. In both diseases, activated microglia were observed [11-14]. In Alzheimer's disease, microglial activation was observed in entorhinal, temporoparietal, and cingulate cortex [12]. In schizophrenia, the significant number of the microglia was observed in hippocampus [13] 
and total grey matter [14]. Thus, microglial activation might be closely related to the pathogenesis of these diseases. Moreover, yokukansan has been shown to have therapeutic effects on microglial cells in vivo [15-17]. For example, yokukansan protects hippocampal neurons from cerebral ischemia through anti-inflammatory effects on microglia [16]. In another study, yokukansan was found to promote hippocampal neurogenesis through suppression of activated microglial cells in Gunn rats [17]. However, whether or not the effects of yokukansan on microglial cells prevent or reduce the progression of the demyelination observed in MS remains unknown.

Cuprizone-induced demyelination is often used to mimic the pathology of human MS [18, 19]. Unlike in other experimental animal models of demyelination, cuprizone, a copper chelator, induces demyelination in the corpus callosum without affecting the blood-brain barrier [18, 20, 21]. In this model, inflammatory cells from the peripheral circulation are rarely observed; therefore, microglial cells provide the major source of inflammatory cytokines and pro-oxidant molecules [22, 23]. Thus, cuprizone-induced demyelination provides a good model to examine the effects of yokukansan on microglial-cell-mediated demyelination. In this study, we investigated the effects of yokukansan on cuprizone-induced demyelination in mouse models and in LPS-stimulated BV2 cells, a murine microglial cell line.

\section{Materials and Methods}

\section{Yokukansan}

Yokukansan is a dry powder composed of extracts of seven herbs: Atractylodes lancea Rhizome, Poria sclerotium, Cnidium rhizome, Uncaria Hook, Japanese Angelica Root, Bupleurum Root and Glycyrrhiza, that are native to Japan, and was supplied by Tsumura \& Co. (Tokyo, Japan). Yokukansan was dissolved in distilled water. In the in vivo experiments, yokukansan was administered orally on a daily basis for 2 weeks at a dose of $1.0 \mathrm{~g} / \mathrm{kg}$ of body weight, which was selected based on the findings of previous studies [17, 24].

\section{Animals}

All experimental protocols were carried out in accordance with the "Principles of laboratory animal care" guidelines provided by the National Institutes of Health, USA, regarding the care and use of animals for experimental procedures, and all experimental protocols were approved by the Institutional Animal Care and Use Committee of Asahikawa Medical University. Every effort was made to minimize animal suffering and to reduce the number of mice used. Female
C57BL/6 mice were obtained from Sankyo Labo Service Corporation, INC, (Tokyo, Japan).

Mouse chow containing $0.2 \%$ cuprizone (bis-cyclohexanone oxaldihydrazone; Sigma, St. Louis, MO, USA) was custom-synthesized (Oriental Yeast Co. LTD, Chiba, Japan). To induce demyelination in adult female C57BL/6 mice ( $n=5-9$ animals per experimental group), animals were fed chow containing $0.2 \%$ cuprizone for a duration of 2-4 weeks, as previously described [25-27]. To evaluate the effects of yokukansan, mice were divided into five groups: normal diet with water, normal diet with yokukansan, 2 weeks of cuprizone-supplemented diet, 4 weeks of cuprizone-supplemented diet with water and 4 weeks of cuprizone-supplemented diet with yokukansan. Either water or water containing yokukansan $(1.0 \mathrm{~g} / \mathrm{kg}$ of body weight) was administered orally by $1 \mathrm{ml}$ syringe with a stainless steel gavage needle on a daily basis for the last 2 weeks of the cuprizone administration period. (Fig. 1).

\section{Histochemistry \& Immunohistochemistry}

Mice ( $n=5-9$ animals per experimental group) were deeply anesthetized using medetomidine $(0.3 \mathrm{mg} / \mathrm{kg}$, Nihon Zenyaku Kogyo, Fukushima, Japan), midazolam $(4.0 \mathrm{mg} / \mathrm{kg}$, Sando, Tokyo, Japan), and butorphanol (5.0 mg/kg, Meiji Seika Pharma, Tokyo, Japan), then perfused with phosphatebuffered saline solution (PBS) followed by $4 \%$ paraformaldehyde in $0.1 \mathrm{M}$ phosphate buffer ( $\mathrm{PB}, \mathrm{pH}$ 7.4). Mouse brains were removed and immersed in the same fixative solution overnight at $4{ }^{\circ} \mathrm{C}$. Mouse brains were cryoprotected using $0.1 \mathrm{M} \mathrm{PB}$ containing $30 \%$ sucrose for 2 days at $4{ }^{\circ} \mathrm{C}$ and then frozen in OCT compound. Frozen $20-\mu \mathrm{m}$-thick sections were cut from their tissue blocks using a cryostat. To assess the extent of demyelination, luxol fast blue (LFB) staining was performed. Sections were rehydrated in $95 \%$ ethanol and immersed in the $0.01 \%$ LFB solution overnight at $55^{\circ} \mathrm{C}$. Sections were then differentiated using a $0.5 \%$ lithium carbonate and $70 \%$ ethanol solution. For immunohistochemistry, rabbit polyclonal anti-Iba- 1 antibody (1:1000, Wako, Osaka, Japan), mouse monoclonal anti-CD68 antibody (1:50, Dako, Santa Clara, CA, USA), goat polyclonal antiIL-1 $\beta$ antibody (1:1000, R \& D Systems, Minneapolis, MN, USA), mouse monoclonal anti-CNPase antibody (1:1000, Sigma, St. Louis, MO, USA), rat monoclonal anti-myelin basic protein (MBP) antibody (1:1000, Abcam, Cambridge, UK), mouse monoclonal anti-glial fibrillary acidic protein (GFAP) antibody (1:2000, Sigma, St. Louis, MO, USA) or rabbit polyclonal anti-NG2 antibody (1:1000, Millipore, Bedford, MA, USA) were used. For immunohistochemistry for CD68, sections were pretreated with Enzyme Pretreatment Kit BOND (Leica, Welzlar, Germany) for 5 min. For immunohistochemistry for CNPase, sections were pretreated with sodium citrate buffer ( $\mathrm{pH} 7.0)$ at $100{ }^{\circ} \mathrm{C}$ for $2 \mathrm{~min}$. 
Fig. 1 Experimental scheme. Six-week-old female C57BL/6 mice were fed with chow either supplemented with or without $0.2 \%$ cuprizone for 4 weeks. After 2 weeks, mice received either $1.0 \mathrm{~g} / \mathrm{kg}$ body weight of yokukansan per day, dissolved in water, or water for another 2 weeks

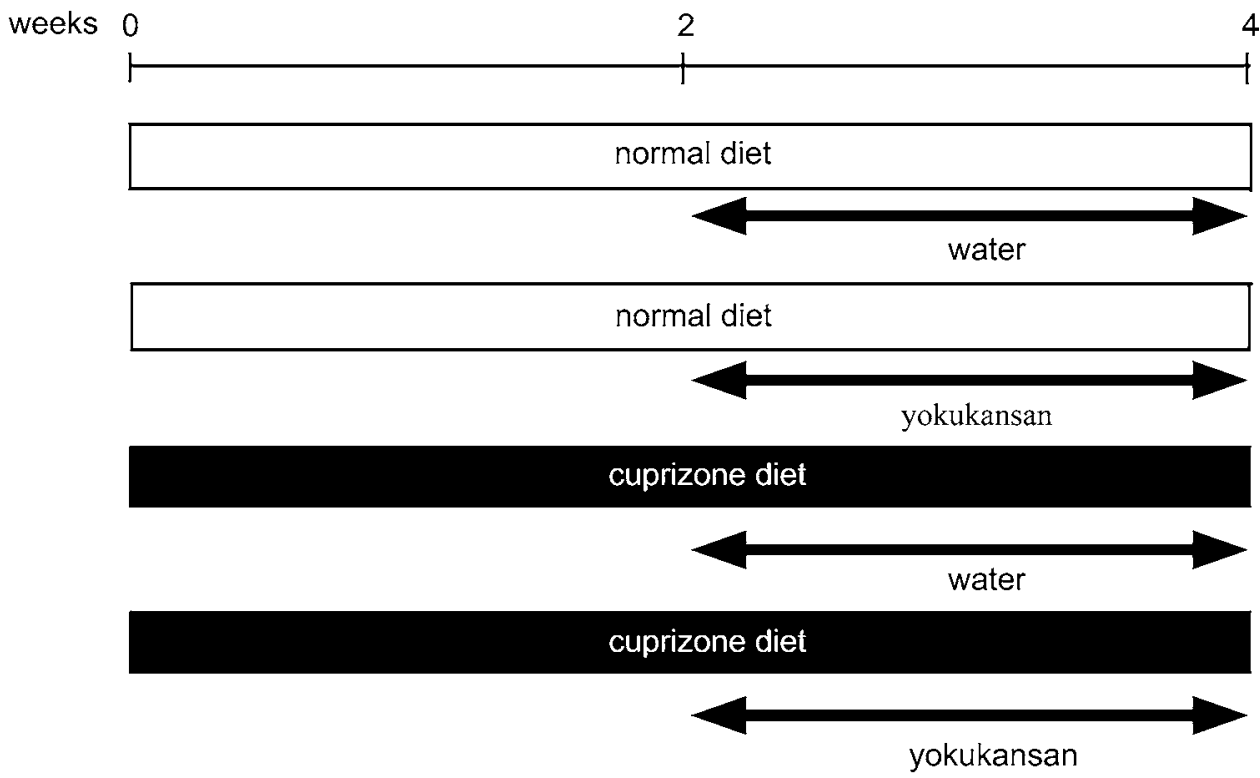

The sections were blocked with a solution containing 5\% bovine serum albumin and $0.3 \%$ triton-X-100 for $60 \mathrm{~min}$ and then incubated with the various primary antibodies at $4{ }^{\circ} \mathrm{C}$ overnight. Sections were then incubated with either Alexa-488-conjugated or Alexa-594-conjugated secondary antibodies (1:1000, Molecular probes, Eugene, OR, USA) followed by nuclear staining with 4',6-diamidino-2-phenylindole (DAPI, Sigma, St. Louis, MO, USA). Sections were then imaged using a confocal laser scanning microscope (Olympus, Tokyo, Japan), and images were analyzed using FV1000-D image analysis software (Olympus, Tokyo, Japan). We then counted the number of microglial cells, astrocytes, oligodendrocyte-progenitor cells (OPCs) and oligodendrocytes that stained positive with either antiIba-1, anti-GFAP, anti-NG2 or anti-CNPase antibodies and/ or DAPI.

\section{BV2 Cell Culture}

BV2 cells, an immortalized murine microglial cell line [28], were cultured in Dulbecco's minimum essential medium supplemented with $10 \%$ fetal calf serum (FCS). BV2 cells were stimulated with $1 \mu \mathrm{g} / \mathrm{ml}$ lipopolysaccharide (LPS) (Sigma, St. Louis, MO, USA). BV2 cells were also treated with yokukansan $(0-500 \mu \mathrm{g} / \mathrm{ml})$ or the equivalent volume of distilled water followed by incubation at $37{ }^{\circ} \mathrm{C}$ for $24 \mathrm{~h}$. Doses of yokukansan were selected on the basis of data from previous in vitro reports $[29,30]$.

\section{Western Blotting}

BV2 cells were homogenized in radioimmunoprecipitation buffer by repeat pipetting for a total of 10 times using a 26-gauge needle attached to a $1-\mathrm{ml}$ syringe. Homogenates were then centrifuged at $15,000 \times g$ for $10 \mathrm{~min}$. The protein concentration of the supernatant was determined using a detergent-compatible protein assay kit (Bio-Rad, Hercules, CA, USA). We boiled $60 \mu \mathrm{g}$ of each protein sample in a sample buffer at $95{ }^{\circ} \mathrm{C}$ for $5 \mathrm{~min}$. These proteins were then separated using $12.5 \%$ sodium dodecyl sulfate-polyacrylamide gel electrophoresis (SDS-PAGE). Proteins were then transferred to an Immobilon-P membrane (Millipore, Bedford, MA, USA). Membranes were then incubated with either rat monoclonal anti-interleukin-1 $\beta$ antibody $(1: 100$; R \& D Systems, Minneapolis, MN, USA), mouse monoclonal anti-iNOS antibody (1:500; BD Biosciences, Franklin Lakes, NJ, USA), or mouse monoclonal anti-glyceraldehyde 3-phosphate dehydrogenase (GAPDH) antibody (1:2500; Ambion, Austin, TX, USA) overnight at $4{ }^{\circ} \mathrm{C}$. Membranes were then incubated with horseradish-peroxidase-linked anti-rat mouse IgG secondary antibodies (1:500-1000; GE Healthcare, Piscataway, NJ, USA) at room temperature for 60-90 min. Following labelling of protein bands using an Amersham ECL select kit (GE Healthcare, Piscataway, NJ, USA), data were acquired using Luminescent Image Analyzer LAS-3000 multi color (FUJIFILM, Tokyo, Japan). The densitometric analysis was performed using ImageJ analysis software (National Institutes of Health, Bethesda, MD, USA) and standardized relative to the density of staining of the GAPDH bands.

\section{Quantitative RT-PCR (qRT-PCR)}

Total RNA was extracted from BV2 cells using TRIzol (Life Technologies, Carlsbad, CA, USA) and cDNA was synthesized using AMV reverse transcriptase according to 
manufacturer's instructions (Promega, Madison, WI, USA). The following PCR primers were used: IL- $1 \beta$ sense primer, 5'-CTCCATGAGCTTTGTACAAGG-3'; IL- $1 \beta$ antisense primer, 5'-TGCTGATGTACCAGTTGGGG-3'; inducible nitric oxide synthase (iNOS) sense primer, $5^{\prime}$-TTGGTGTTT GGGTGCCGGC-3'; iNOS antisense primer, 5'-CCATAG GAAAAGACTGCACCGAAG-3'; GAPDH sense primer, 5'-CTACATGGTCTACCTGTTCCAG-3'; GAPDH antisense primer, 5'-AGTTGTCATGGATGACCTTGG-3'. PCR cycling conditions consisted of an initial $5 \mathrm{~min}$ at $96^{\circ} \mathrm{C}$, followed by 50 cycles of $30 \mathrm{~s}$ at $96{ }^{\circ} \mathrm{C}, 30 \mathrm{~s}$ at $60-63{ }^{\circ} \mathrm{C}$, and $30 \mathrm{~s}$ at $72^{\circ} \mathrm{C}$. For qRT-PCR, we used LightCycler FastStart DNA MasterPLUS SYBR Green I (Roche, Mannheim, Germany). We used relative quantification method according to the manufacturer's protocol.

\section{Data Analysis}

For semi-quantitative analysis, intensities of MBP immunoreactivity were measured using ImageJ software. The number of microglial cells, astrocytes, OPCs and oligodendrocytes staining positive for their respective markers that were located completely around the DAPI-stained nucleus were counted and recorded. These analyses were conducted by an investigator blinded to the treatment groups to omit the possibility of bias. A one-way ANOVA, followed by a Tukey-Kramer test was used to compare differences between mean values for each group. Differences were deemed statistically significant if they exceeded the $95 \%$ confidence interval $(P<0.05)$.

\section{Results}

\section{Yokukansan Reduces Cuprizone-Induced Demyelination}

Mice were fed chow supplemented with or without $0.2 \%$ cuprizone for a duration of 4 weeks to induce demyelination. In the last 2 weeks of cuprizone treatment, mice also received either orally administered daily yokukansan or distilled water at doses of $1.0 \mathrm{~g} / \mathrm{kg}$ body weight per day (Fig. 1). The extent of demyelination was evaluated using LFB staining (Fig. 2a-d) and immunostaining for MBP (Fig. 2e-h, j) in the corpora callosa. The extent of demyelination and glial responses were evaluated in the corpora callosa depicted in the red-boxed area of Fig. 2i. The corpora callosa of control mice had an even distribution of LFB staining (Fig. 2a), and administration of yokukansan to mice receiving their normal diet had no significant effect on the distribution of LFB staining in the corpora callosa of these animals (Fig. 2b). The corpora callosa were thinner in mice exposed to cuprizone, relative to those of control mice, and areas that did not stain with LFB were observed (Fig. 2c). Administration of yokukansan resulted in the recovery of uniform distribution of LFB staining in cuprizone-fed mice (Fig. 2d), and similar changes in the distribution of MBP immunoreactivity were observed in the corpora callosa of mice exposed to cuprizone (Fig. 2e-h). Administration of yokukansan to mice fed a normal diet resulted in no significant differences in MBP immunoreactivity (Fig. 2f). However, the corpora callosa of cuprizone-fed mice had significantly reduced MBP immunoreactivity (Fig. 2g), and this decreased immunoreactivity was reduced by administration of yokukansan (Fig. 2h). The intensity of MBP immunofluorescence was significantly reduced by cuprizone treatment compared with that of control mice $(0.56 \pm 0.04$ vs. $1.00 \pm 0.05 ; P<0.01)$ or yokukansan-treated mice fed a normal diet $(0.56 \pm 0.04$ vs. $0.92 \pm 0.10 ; P<0.01)$. However, administration of yokukansan to cuprizone-fed mice significantly reduced these decrease of intensity of MBP immunoreactivity compared with that of control-treated cuprizone-fed mice $(0.83 \pm 0.06$ vs. $0.56 \pm 0.04 ; P=0.021$ ) (Fig. $2 \mathrm{j}$ ).

\section{Yokukansan Reduced the Accumulation of Microglial Cells in the Corpora Callosa of Cuprizone-fed Mice}

Cuprizone-induced demyelination is closely related to the accumulation and activation of inflammatory responses in microglial cells [20, 31-33]. Thus, we examined whether the accumulation of microglial cells induced by cuprizone was affected by administration of yokukansan. Microglia in the corpora callosa of control mice were evenly distributed $\left(82.2 \pm 14.2\right.$ per $\mathrm{mm}^{2}$ ) (Fig. 3a, f) and administration of yokukansan had no significant effect on the number of Iba-1-positive microglia $\left(94.7 \pm 13.0\right.$ per $\mathrm{mm}^{2}$ ) (Fig. 3b, $\mathrm{g}, \mathrm{k}$ ). Both mice exposed to 2 weeks of cuprizone, just before administration of water or yokukansan, and 4 weeks of cuprizone had significantly increased numbers of Iba1-positive microglial cells in the corpora callosa compared with control mice $(439.8 \pm 55.0$ vs. $82.2 \pm 14.2,623.3 \pm 54.8$ vs. $82.2 \pm 14.2$ per $\mathrm{mm}^{2} ; P<0.01$ for both comparisons) (Fig. 3c, d, h, i, k). Administration of yokukansan to cuprizone-fed mice significantly decreased the number of Iba1-positive microglial cells compared with that of cuprizonefed mice $\left(415.9 \pm 70.6\right.$ vs. $623.3 \pm 54.8$ per $\left.\mathrm{mm}^{2} ; P=0.022\right)$ (Fig. 3e, j, k). These results indicate that yokukansan suppresses the accumulation of Iba-1-positive microglia and reduces cuprizone-induced demyelination.

\section{Yokukansan Reduced Both the Number of CD68-positive Microglia and IL-1ß-positive Microglia in the Corpora Callosa of Cuprizone-fed Mice}

To examine the number of activated microglia in the corpus callosum of cuprizone-fed mice, we performed 

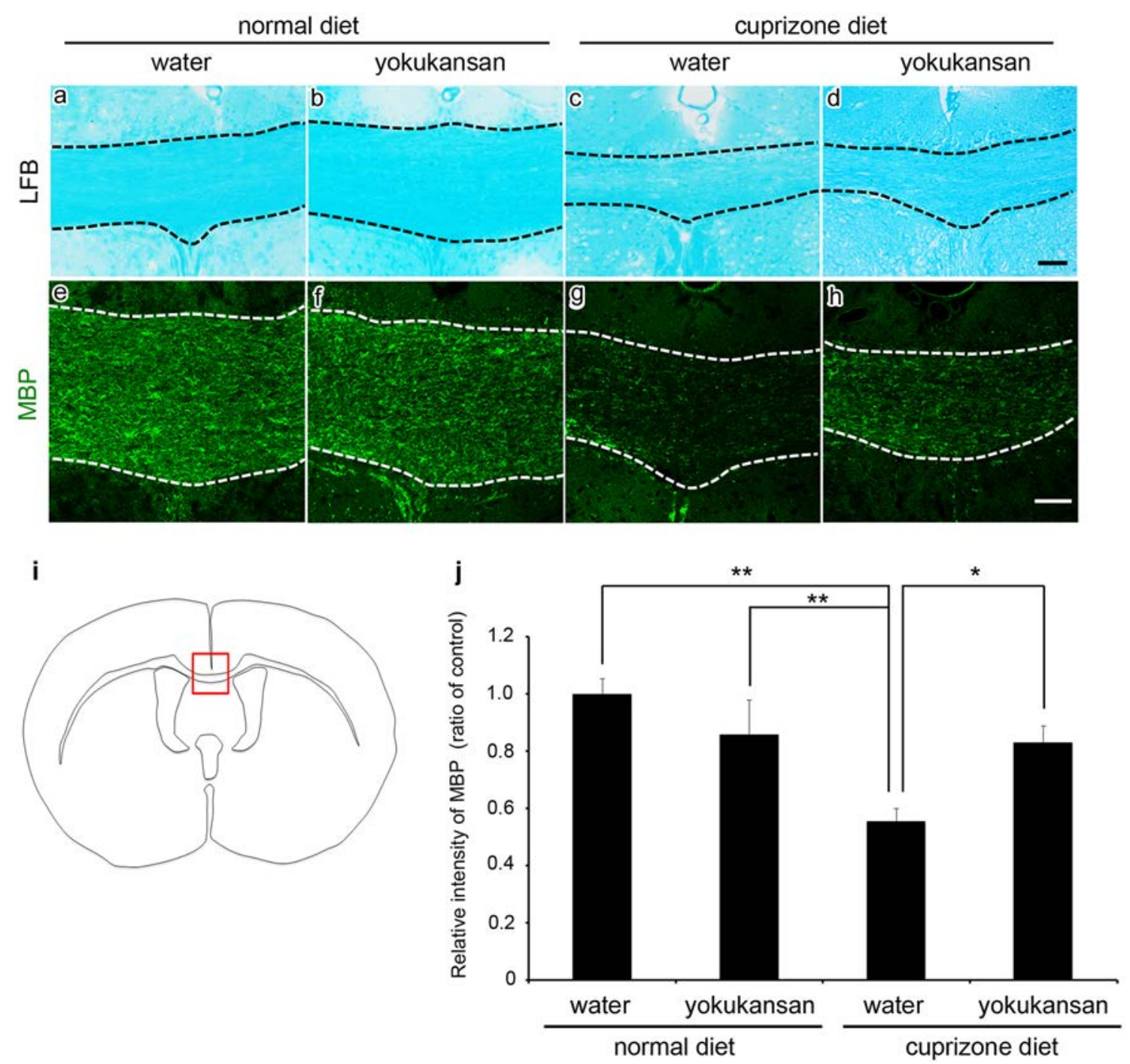

Fig. 2 Yokukansan reduced cuprizone-induced demyelination in the corpus callosum. We used 6 weeks age of C57BL/6 mice in these experiments. $\mathbf{a}-\mathbf{h}$ In each group, the extent of demyelination was examined in the corpora callosa using LFB staining and MBP immunohistochemistry. Representative images of LFB staining (a-d) and MBP immunoreactivity $(\mathbf{e}-\mathbf{h})$ are shown. Scale bar $=50 \mu \mathrm{m}$. i All

immunohistochemistry for CD68. Administration of yokukansan to mice fed a normal diet resulted in no significant differences in the number of CD68+Iba-1+ microglia. The number of CD68+Iba-1+ microglia significantly increased in the corpora callosa in mice exposed to 4 weeks of cuprizone compared with both control mice and mice exposed to 2 weeks of cuprizone $(416.3 \pm 48.3$ vs. $16.1 \pm 4.9,416.3 \pm 48.3$ vs. $143.8 \pm 11.3$ per $\mathrm{mm}^{2} ; P<0.01$ for both comparisons) (Fig. 4a-d). While administration of yokukansan significantly reduced the number of CD68+Iba-1+ microglia in the corpora callosa compared with cuprizone-fed mice with water $\left(249.7 \pm 18.6\right.$ vs. $416.3 \pm 48.3$ per $\left.\mathrm{mm}^{2} ; P<0.01\right)$, still the number of that significantly increased compared images used to measure the extent of cuprizone-induced demyelination were taken of the area outlined by the red box. $\mathbf{j}$ Semi-quantitative assessments of the intensity of MBP immunoactivity were made using raw data obtained from such images. All data presented in the figures are the mean \pm SEM of data from 6 to 9 animals. $* * P<0.01$, $* P<0.05$

to mice exposed to 2 weeks of cuprizone $(249.7 \pm 18.6$ vs. $143.8 \pm 11.3$ per $\left.\mathrm{mm}^{2} ; P<0.05\right)$.

Next we performed immunohistochemistry for IL-1 $\beta$. Administration of yokukansan to mice fed a normal diet resulted in no significant differences in the number of IL- $1 \beta+$ Iba- $1+$ microglia. The number of IL- $1 \beta+\mathrm{Iba}-1+$ microglia significantly increased in the corpora callos $a$ in mice exposed to 4 weeks of cuprizone compared with both control mice and mice exposed to 2 weeks of cuprizone $(208.7 \pm 33.8$ vs. $4.8 \pm 3.1,208.7 \pm 33.8$ vs. $72.6 \pm 13.6$ per $\mathrm{mm}^{2} ; P<0.01$ for both comparisons) (Fig. $4 \mathrm{e}-\mathrm{h}$ ). Administration of yokukansan significantly reduced the number of IL- $1 \beta+\mathrm{Iba}-1+$ microglia in the 

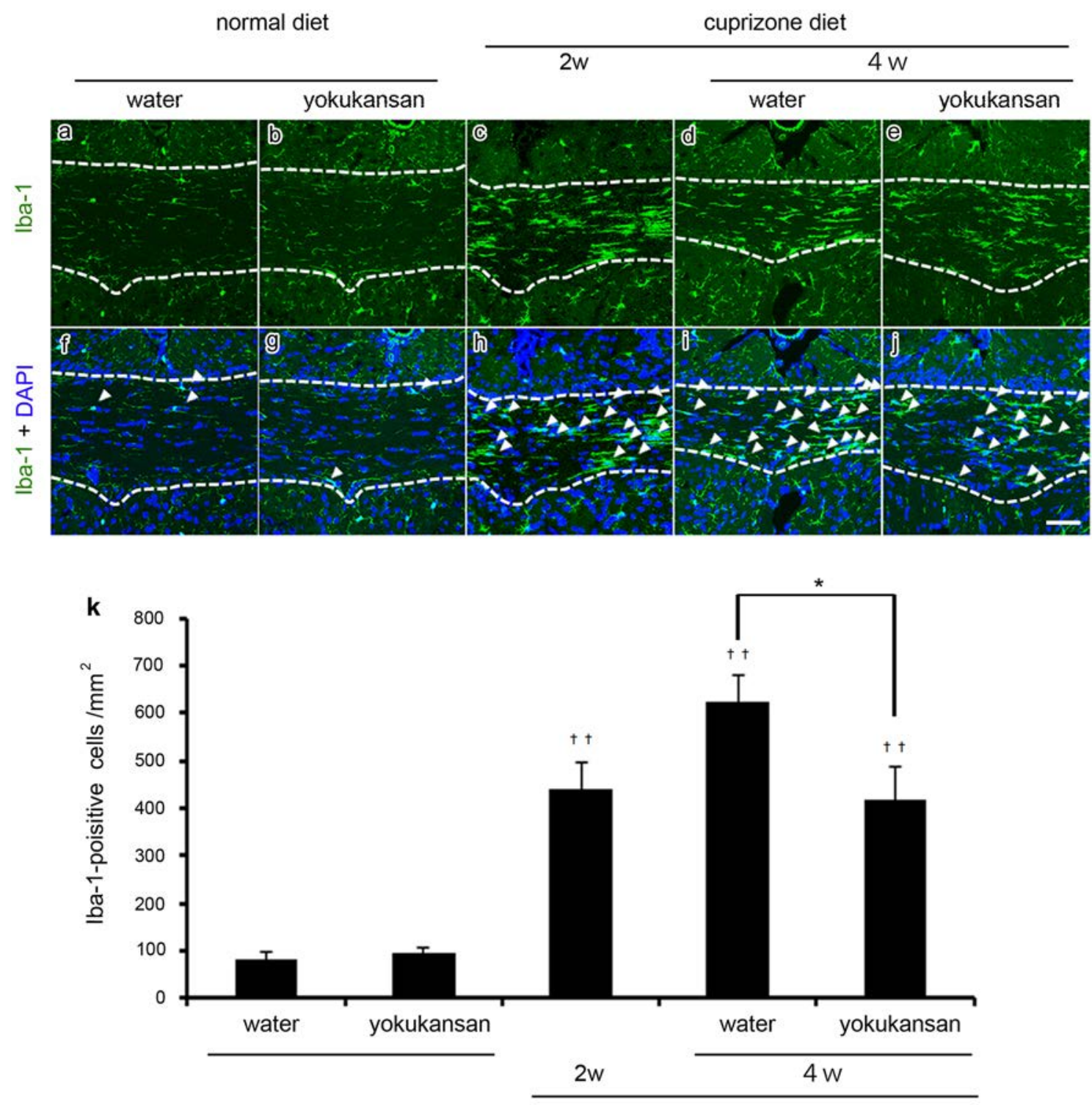

normal diet

cuprizone diet

Fig. 3 Yokukansan reduced the number of Iba-1 positive cells in the corpus callosum. a-j Representative images showing Iba-1 immunoreactivity (green) (a-e) and merged images of Iba-1 and nuclear staining with DAPI (blue) $(\mathbf{f}-\mathbf{j})$. Scale bar $=50 \mu \mathrm{m}$. k Semi-quanti-

corpora callosa compared with cuprizone-fed mice with water $\left(91.9 \pm 12.8\right.$ vs. $208.7 \pm 33.8$ per $\left.\mathrm{mm}^{2} ; P<0.01\right)$.

\section{Yokukansan Affected Astrocytes in the Corpora Callosa of Cuprizone-fed Mice}

We next examined the responses of other glial cells, astrocytes, OPCs and oligodendrocytes, in the corpus callosum to yokukansan. Treatment with yokukansan did not affect the numbers of astrocytes, OPCs or oligodendrocytes compared with those of control mice (Fig. 5i). In mice fed a tative analysis of the number of Iba-1 positive cells in the corpora callosa. Data are presented as the mean \pm SEM from 5 to 9 animals. $* P<0.05,{ }^{\dagger+} P<0.01$ relative to animals fed a normal diet with either water or yokukansan

cuprizone-containing diet, the number of GFAP-positive astrocytes in the corpora callosa increased significantly compared with that of control mice $(366.8 \pm 56.4$ vs. $100.2 \pm 12.4$ per $\mathrm{mm}^{2} ; P<0.01$ ) (Fig. 5c, g). Furthermore, administration of yokukansan to cuprizone-fed mice significantly decreased the number of GFAP-positive astrocytes compared with that of cuprizone-fed mice $(145.7 \pm 22.0$ vs. $366.8 \pm 56.4$ per $\mathrm{mm}^{2} ; P<0.01$ ) (Fig. $\left.5 \mathrm{~d}, \mathrm{~h}, \mathrm{i}\right)$. Furthermore, the number of NG2-positive OPCs was also significantly increased in cuprizone-treated mice compared with control mice $\left(204.3 \pm 20.7\right.$ vs. $108.1 \pm 16.4$ per $\left.\mathrm{mm}^{2} ; P<0.01\right)$. 

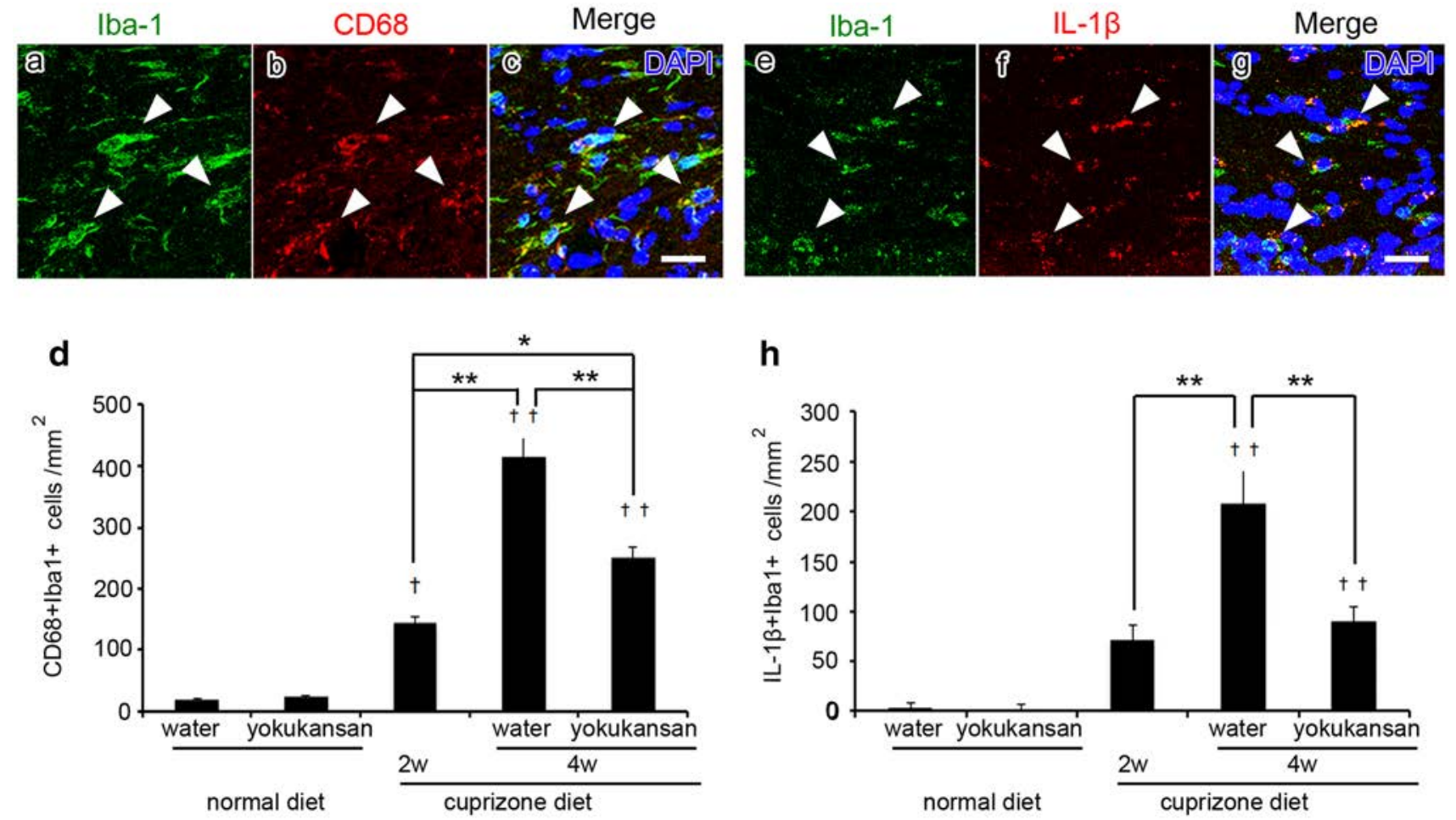

Fig. 4 Yokukansan reduced both the number of CD68-positive and IL-1 $\beta$-positive microglia in the corpus callosum. a-c Representative images showing Iba-1 (green) (a) and CD68 (red) (b) immunoreactivity and merged images of Iba-1, CD68 and nuclear staining with DAPI (blue) (c). d Semi-quantitative analysis of the number of CD68 and Iba-1 double positive cells in the corpora callosa. e-g Representative images showing Iba-1 (green) (e) and IL-1 $\beta$ (red) (f) immu-

Administration of yokukansan to cuprizone-fed mice had no significant effect on the number of OPCs in the corpora callosa (Fig. 5i). In mice fed a cuprizone-containing diet, the number of CNPase-positive oligodendrocytes decreased significantly compared with that of control mice $\left(200.0 \pm 31.1\right.$ vs. $446.4 \pm 62.7$ per $\left.\mathrm{mm}^{2} ; P<0.01\right)$. Administration of yokukansan to cuprizone-fed mice significantly increased the number of oligodendrocytes $(405.8 \pm 36.7$ vs. $200.0 \pm 31.1$ per $\left.\mathrm{mm}^{2} ; P<0.05\right)$ (Fig. $\left.5 \mathrm{i}\right)$.

\section{Yokukansan Treatment Attenuated the Expression of IL-1ß and iNOS in LPS-stimulated BV2 Cells}

BV2 cells, a murine microglial cell line, were used to investigate the direct effects of yokukansan on microglial cells. No significant alterations in IL- $1 \beta$ or iNOS expression were observed in non-LPS-stimulated BV2 cells exposed to either 100 or $500 \mu \mathrm{g} / \mathrm{ml}$ yokukansan compared with controls. LPS stimulation did not induce morphological changes in BV2 cells (data not shown). However, LPS stimulation resulted in a significant increase in both IL- $1 \beta$ and iNOS mRNA compared with controls $(1.20 \pm 0.18$ vs. $0.03 \pm 0.01$, and

noreactivity and merged images of Iba-1, IL- $1 \beta$ and nuclear staining with DAPI (blue) (g). h Semi-quantitative analysis of the number of IL-1 $\beta$ and Iba- 1 double positive cells in the corpora callosa. Scale bar $=20 \mu \mathrm{m}$. Data are presented as the mean \pm SEM from 5 to 9 animals. ${ }^{* *} P<0.01, * P<0.05,{ }^{+\dagger} P<0.01,{ }^{\dagger} P<0.05$ relative to animals fed a normal diet with either water or yokukansan

$1.37 \pm 0.16$ vs. $0.03 \pm 0.01$, respectively; $P<0.01$ for both comparisons). (Fig. 6a, b). Treatment of LPS-stimulated BV2 cells with $100 \mu \mathrm{g} / \mathrm{ml}$ yokukansan tended to reduce the expression of IL- $1 \beta$ and iNOS mRNA. Furthermore, treatment with $500 \mu \mathrm{g} / \mathrm{ml}$ yokukansan significantly reduced both IL-1 $\beta$ and iNOS mRNA levels compared with those of LPS-stimulated controls $(0.39 \pm 0.08$ vs. $1.20 \pm 0.18$, and $0.73 \pm 0.09$ vs. $1.37 \pm 0.16 ; P<0.01$ for both comparisons) (Fig. 6a, b). Expression of IL-1 $\beta$ and iNOS proteins was then investigated using western blotting (Fig. 6c). Similar to data obtained using qRT-PCR, semi-quantitative densitometry analysis revealed no significant effects of yokukansan on unstimulated BV2 cells; however, stimulation with LPS significantly increased the production of both IL- $1 \beta$ and iNOS compared with unstimulated controls $(0.19 \pm 0.06$ vs. $1.00 \pm 0.15$, and $0.16 \pm 0.05$ vs. $1.02 \pm 0.16$, respectively; $P<0.01$ for both comparisons) (Fig. 6a, b). Furthermore, $100 \mu \mathrm{g} / \mathrm{ml}$ yokukansan tended to reduce IL- $1 \beta$ and iNOS production compared with that of LPS-stimulated controls, and $500 \mu \mathrm{g} / \mathrm{ml}$ yokukansan significantly reduced IL- $1 \beta$ and iNOS expression compared with LPS-stimulated controls $(0.40 \pm 0.10$ vs. $1.00 \pm 0.15$, and $0.37 \pm 0.09$ vs. $1.02 \pm 0.16$, 


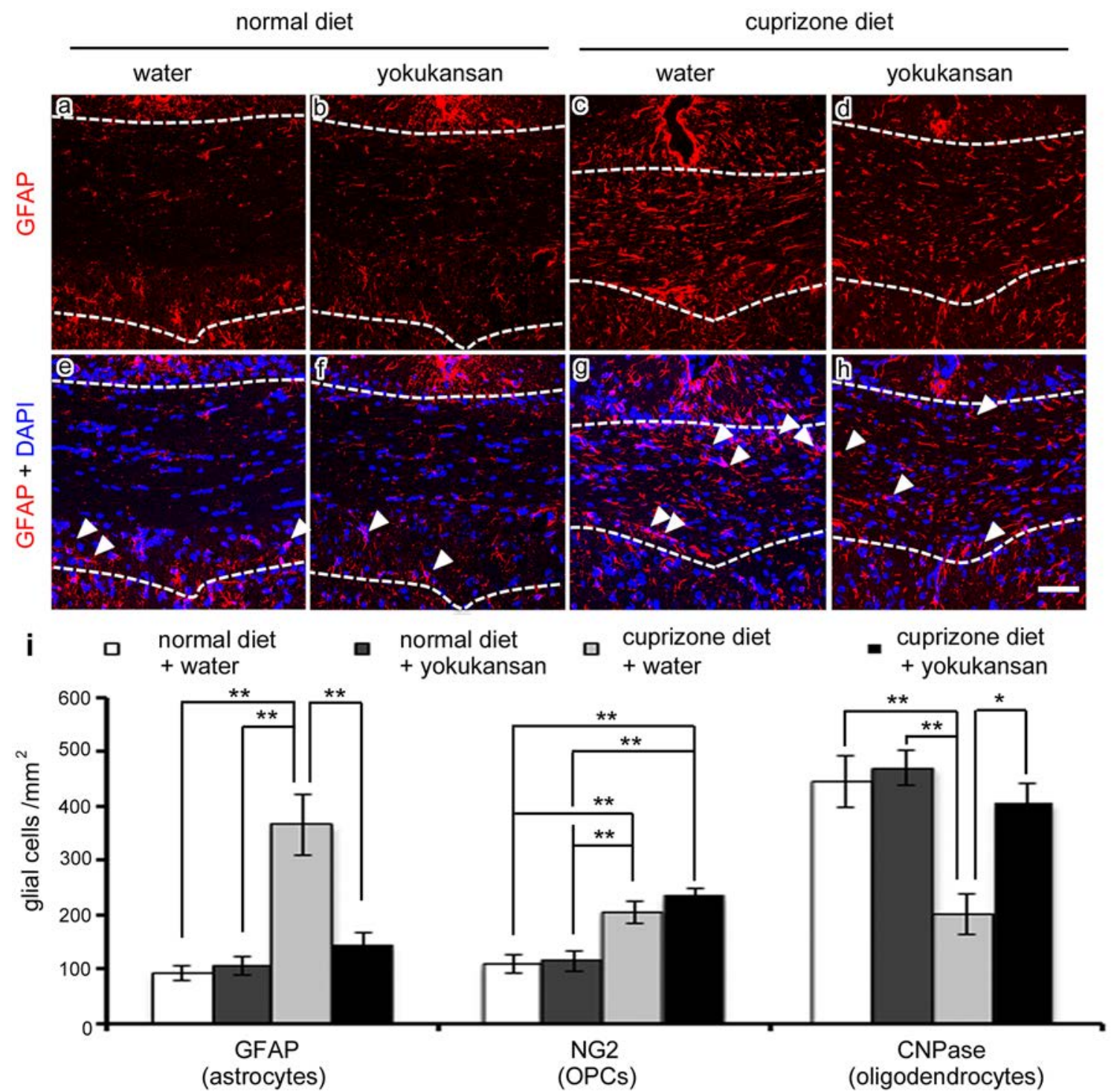

Fig. 5 Yokukansan affected other glial cells in the corpus callosum. a-h Representative images of GFAP immunoreactivity (red) (a-d) and merged views of GFAP, and DAPI immunoreactivity (e-

respectively; $P<0.01$ for both values) (Fig. 6c, d, e). These data indicate that $500 \mu \mathrm{g} / \mathrm{ml}$ yokukansan has anti-inflammatory effects on LPS-stimulated BV2 cells.

\section{Discussion}

Yokukansan has been used to treat various diseases such as Alzheimer's disease and schizophrenia [6-10]. However, whether or not yokukansan is able to reduce demyelination in the CNS remains unknown. Here, we demonstrated for the first time that yokukansan reduces cuprizone-induced demyelination in the corpus callosum and suppresses the h). Scale bar $=50 \mu \mathrm{m}$. (i) Semi-quantitative analysis of the number of GFAP, NG2, or CNPase-positive cells. Data are presented as the mean \pm SEM from 6 to 9 animals. $* * P<0.01, * P<0.05$

expression of IL- $1 \beta$ and iNOS mRNA and protein in LPSstimulated BV2 cells, a murine microglial cell line.

While administration of cuprizone diet induces demyelination, withdrawal of cuprizone-feeding promotes remyelination. In a previous study, geissoschizine methyl ether (GM) in the Uncaria Hook, a constituent of yokukansan, was given for 2 weeks after withdrawal of cuprizone-feeding. Then the administration of GM significantly promoted remyelination in the medial prefrontal cortex [34]. Thus, yokukansan might promote remyelination in the corpus callosum in addition to suppression of demyelination. In a previous study, investigators observed spontaneous remyelination in the corpus callosum after 5-6 weeks of cuprizone administration [19]. In fact, while more than $90 \%$ of axons 

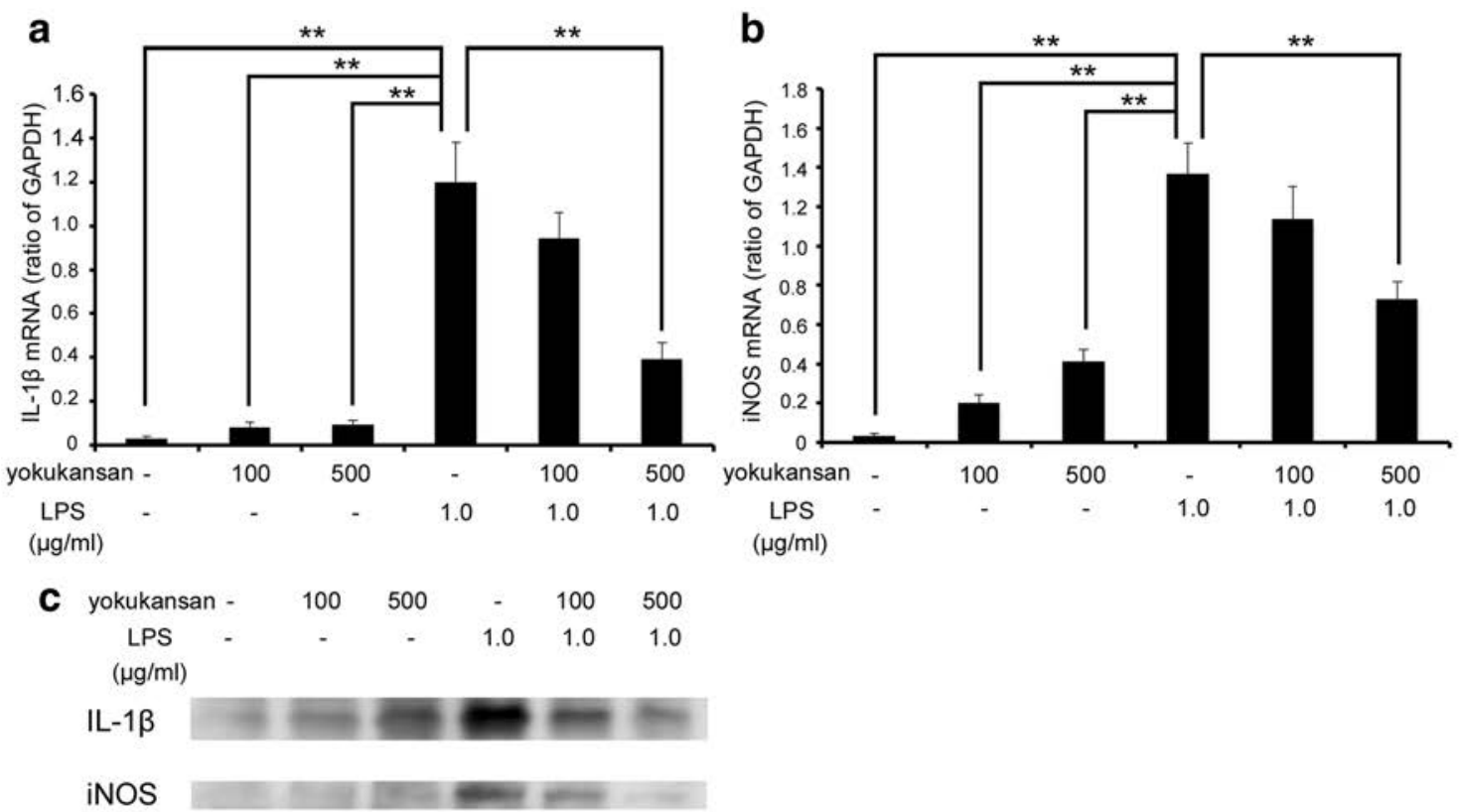

GAPDH
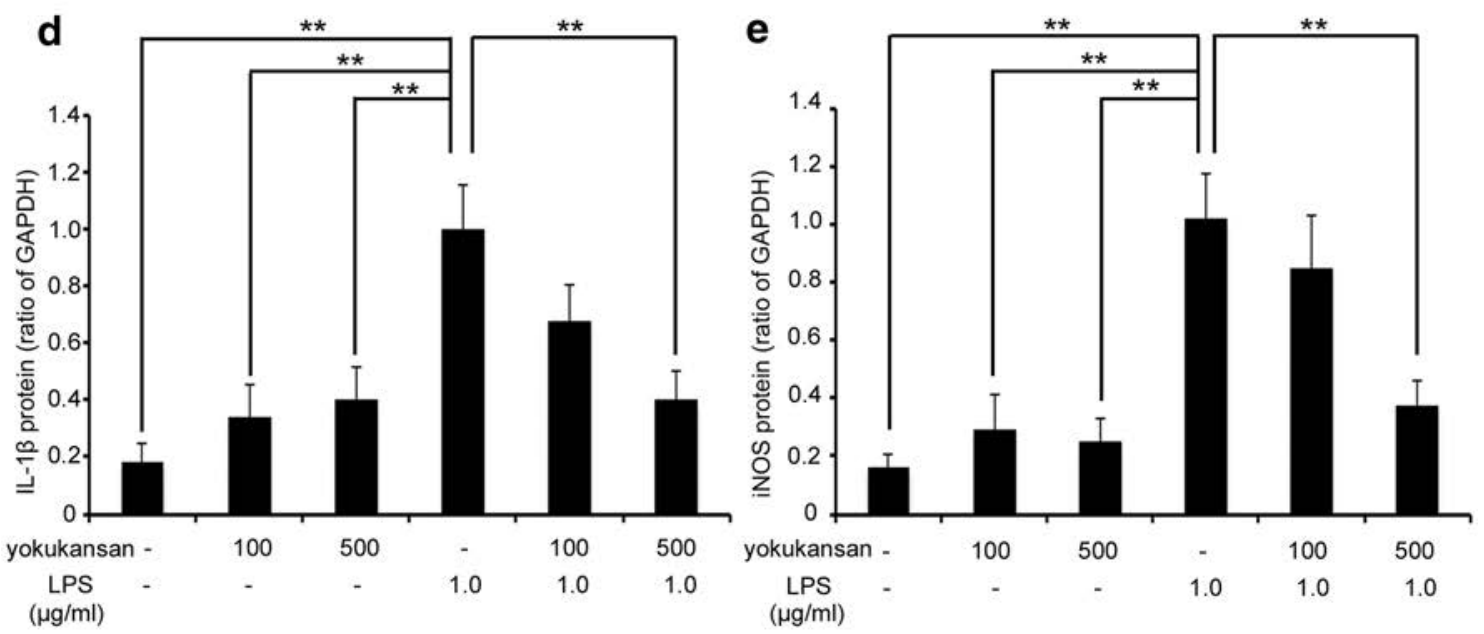

Fig. 6 Yokukansan reduced levels of IL-1 $\beta$ and iNOS mRNA and protein in LPS-stimulated BV2 cells. a Expression of IL- $1 \beta$ mRNA in LPS-stimulated or unstimulated BV2 cells in the presence or absence of yokukansan (100 or $500 \mu \mathrm{g} / \mathrm{ml})$. b Expression of iNOS mRNA in LPS-stimulated or unstimulated BV2 cells in the presence

were demyelinated by 4-5 weeks of oral cuprizone administration, about $50 \%$ of axons were spontaneously remyelinated by 6 weeks of administration [19]. In this process, NG2-positive OPCs started to proliferate after 4 weeks of cuprizone administration, and then these OPCs differentiated into mature oligodendrocytes after 6 weeks of cuprizone administration [35]. In this study, we focused on the demyelinating phase, during which OPCs proliferate, but not differentiate in the corpus callosum. Our results showed or absence of yokukansan (100 or $500 \mu \mathrm{g} / \mathrm{ml})$. c Western blotting for IL-1 $\beta$, iNOS and GAPDH proteins. Semi-quantitative densitometric analysis of (d) IL-1 $\beta$ and (e) iNOS levels. Data are presented as the mean \pm SEM nine independent experiments. ${ }^{*} P<0.01, * P<0.05$

that yokukansan had no significant effect on the number of NG2-positive OPCs. Therefore, the effect of yokukansan in the demyelination phase may not be to promote remyelination but to inhibit demyelination.

During cuprizone-induced demyelination, inflammatory responses of microglial cells promote demyelination in the corpus callosum [18, 20, 33]. Data from several studies demonstrate that suppression of microglial accumulation reduces cuprizone-induced demyelination $[20$, 
32]. Furthermore, accumulating evidence suggests that treatment with yokukansan suppresses microglial activity. Yokukansan has been shown to suppress microglial activity in Gunn rats, an animal model of schizophrenia [17]. In another report, yokukansan reduced the number of activated microglial cells and suppressed the inflammation associated with cerebral ischemia in gerbils [16]. Our results indicate that yokukansan reduces the number of microglia activated by cuprizone-induced demyelination and provides further evidence of the anti-inflammatory effects of yokukansan on microglia. Therefore, we investigated whether or not yokukansan has direct anti-inflammatory effects in vitro using BV2 cells, an immortalized murine microglial cell line [28]. Although LPS-stimulated BV2 cells did not show morphological changes, BV2 cells retain inducible secretory functions such as inflammatory cytokines and pro-oxidant molecules [28, 36]. In fact, $90 \%$ of the inflammatory gene expressions induced by LPS in BV2 cells was also found in primary microglia [37]. Thus, using LPS-stimulated BV2 cells are suitable to investigate inflammatory responses of microglia in vitro. From in vivo and in vitro results, we demonstrated that yokukansan has anti-inflammatory effects on microglia in the corpus callosum during cuprizone-induced demyelination. However, it is possible that yokukansan has some direct effects on oligodendrocytes and oligodendrocyte progenitor cells. Therefore, further studies to elucidate the effect of yokukansan are needed in future.

Treatment with yokukansan reduced the expression of IL-1 $\beta$ and iNOS in LPS-stimulated BV2 cells, and yokukansan significantly reduced IL- $1 \beta$-positive microglia in the corpora callosa during cuprizone-induced demyelination. In other studies, administration of minocycline resulted in suppression of microglial activation, with reduced expression of IL-1 $\beta$ and iNOS in the CNS [38] and reduction of cuprizone-induced demyelination in the corpus callosum $[33,39]$. Decreased IL-1 $\beta$ levels in vivo may also reflect a reduction in the activation of astrocytes because IL-1 $1 \beta$ has a key role in proliferation of astrocytes in vitro and in vivo $[40,41]$. IL- $1 \beta$ is mainly produced by microglia in the CNS $[22,42]$, and while microglial cells started to appear after 1 week of cuprizone exposure, astrocytes began to increase after 3 weeks of cuprizone treatment [31]. Taken together, cuprizone exposure may induce microglial activation, and IL-1 $\beta$ from microglia may proliferate astrocytes in the corpus callosum. Therefore, decreased number of astrocytes in the corpus callosum may at least in part reflect the antiinflammatory effect of yokukansan on microglia.

Yokukansan is composed of extracts of seven herbs. One of the active ingredients, GM in Uncaria Hook, was reported to cross the blood brain barrier in vivo [43] and has psychotropic components. GM distributed in many regions in the brain including the medial prefrontal cortex and promoted recovery from cuprizone-induced demyelination [34]. However, the anti-inflammatory effect of GM in cuprizone-induced demyelination has not been reported and the concentration of GM in the corpus callosum was relatively low [44]. Therefore, some other active ingredients of yokukansan may suppress inflammatory responses in the corpus callosum.

In the current study, yokukansan suppressed the expression of IL- $1 \beta$ and iNOS mRNA and protein in LPS-stimulated BV2 cells in vitro, suggesting that yokukansan affects the transcription of these molecules. In LPS-stimulated BV2 cells, activation of inflammation related kinases (JNK and $\mathrm{p}-38$ ) and transcription factors (c-JUN and NF- $\mathrm{KB}$ ) play roles in producing inflammatory cytokines $[37,45]$. Therefore, yokukansan may repress these pathways. However, it has not been fully identified which active ingredients play critical roles in the anti-inflammatory effect in microglia. Further studies will be required for identification of active ingredients and signal pathways interacted.

For remyelination, clearance of myelin debris is instrumental $[46,47]$. In the current study, yokukansan reduced cuprizone-induced demyelination in the corpora callosa. Administration of yokukansan significantly reduced Iba$1+$ microglia and IL- $1 \beta+\mathrm{Iba}-1+$ microglia in the corpora callosa during cuprizone-induced demyelination. Thus it is considered that yokukansan reduced inflammatory responses and myelin debris may be relatively less compared to cuprizone-fed mice with water. Moreover, although yokukansan significantly reduced the number of phagocytic microglia in cuprizone-fed mice, the number of phagocytic microglia in cuprizone-fed mice with yokukansan still significantly increased compared to that in 2 weeks of cuprizone-fed mice, just before administration of yokukansan. Therefore, yokukansan may retain phagocytic ability of microglia in part. Studies for the effect of yokukansan on remyelination are needed in future.

Inflammatory cytokines and pro-oxidant molecules cause irreversible damage in demyelination in the CNS $[2,4]$. It is possible that yokukansan reduces these inflammatory responses without serious adverse effects [6-10]. Moreover, yokukansan might also promote maturation of oligodendrocytes during the remyelination phase [34]. Yokukansan has the potential to be a novel option to treat MS.

Acknowledgements Yokukansan was provided by Tsumura Research Laboratory, Tsumura \& Co., Ibaraki, Japan. We thank for Dr. Morita, Dr. Yamamoto, Ms. Akiyama, for technical assistance. This work was supported by the Japan Society for the Promotion of Science (JSPS) KAKENHI Grant Number JP 16k07023 and grants from the Akiyama Memorial Foundation (Yoshio Bando). Hua You is supported by the National Natural Science Foundation of China (81670180, 81711540047,81370077 , and 81001220), and the Beijing Nova Program of the Beijing Municipal Science and Technology Commission (Z171100001117091). 


\section{Compliance with Ethical Standards}

Conflict of interest The authors declare that they have no conflict of interest.

\section{References}

1. Hafler DA (2004) Multiple sclerosis. J Clin Invest 113:788-794

2. Grigoriadis N, van Pesch V (2015) A basic overview of multiple sclerosis immunopathology. Eur J Neurol 22:3-13

3. Hauser SL, Doolittle TH, Lincoln R, Brown RH, Dinarello CA (1990) Cytokine accumulations in CSF of multiple sclerosis patients: frequent detection of interleukin-1 and tumor necrosis factor but not interleukin-6. Neurology 40:1735-1739

4. Fischer MT, Sharma R, Lim JL, Haider L, Frischer JM, Drexhage J, Mahad D, Bradl M, van Horssen J, Lassmann H (2012) NADPH oxidase expression in active multiple sclerosis lesions in relation to oxidative tissue damage and mitochondrial injury. Brain 135:886-899

5. Brosnan CF, Cannella B, Battistini L, Raine CS (1995) Cytokine localization in multiple sclerosis lesions: correlation with adhesion molecule expression and reactive nitrogen species. Neurology 45:S16-21

6. Iwasaki K, Satoh-Nakagawa T, Maruyama M, Monma Y, Nemoto M, Tomita N, Tanji H, Fujiwara H, Seki T, Fujii M, Arai H, Sasaki $\mathrm{H}$ (2005) A randomized, observer-blind, controlled trial of the traditional Chinese medicine Yi-Gan San for improvement of behavioral and psychological symptoms and activities of daily living in dementia patients. J Clin Psychiatry 66:248-252

7. Monji A, Takita M, Samejima T, Takaishi T, Hashimoto K, Matsunaga H, Oda M, Sumida Y, Mizoguchi Y, Kato T, Horikawa H, Kanba S (2009) Effect of yokukansan on the behavioral and psychological symptoms of dementia in elderly patients with Alzheimer's disease. Prog Neuropsychopharmacol Biol Psychiatry 33:308-311

8. Miyaoka T, Furuya M, Horiguchi J, Wake R, Hahioka S, Tohyma M, Mori N, Minabe Y, Iyo M, Ueno S, Ezoe S, Murotani K, Hoshino S, Seno H (2015) Efficacy and safety of yokukansan in treatment-resistant schizophrenia: a randomized, double-blind, placebo-controlled trial (a positive and negative syndrome scale, five-factor analysis). Psychopharmacology 232:155-164

9. Furukawa K, Tomita N, Uematsu D, Okahara K, Shimada H, Ikeda M, Matsui T, Kozaki K, Fujii M, Ogawa T, Umegaki H, Urakami K, Nomura H, Kobayashi N, Nakanishi A, Washimi Y, Yonezawa H, Takahashi S, Kubota M, Wakutani Y, Ito D, Sasaki T, Matsubara E, Une K, Ishiki A, Yahagi Y, Shoji M, Sato H, Terayama Y, Kuzuya M, Araki N, Kodama M, Yamaguchi T, Arai H (2017) Randomized double-blind placebo-controlled multicenter trial of Yokukansan for neuropsychiatric symptoms in Alzheimer's disease. Geriatr Gerontol Int 17:211-218

10. Yu CH, Ishii R, Yu SC, Takeda M (2014) Yokukansan and its ingredients as possible treatment options for schizophrenia. Neuropsychiatr Dis Treat 10:1629-1634

11. Su F, Bai F, Zhang Z (2016) Inflammatory cytokines and Alzheimer's disease: a review from the perspective of genetic polymorphisms. Neurosci Bull 32:469-480

12. Cagnin A, Brooks DJ, Kennedy AM, Gunn RN, Myers R, Turkheimer FE, Jones T, Banati RB (2001) In-vivo measurement of activated microglia in dementia. Lancet 358:461-467

13. Doorduin J, de Vries EF, Willemsen AT, de Groot JC, Dierckx RA, Klein HC (2009) Neuroinflammation in schizophrenia-related psychosis: a PET study. J Nucl Med 50:1801-1807
14. van Berckel BN, Bossong MG, Boellaard R, Kloet R, Schuitemaker A, Caspers E, Luurtsema G, Windhorst AD, Cahn W, Lammertsma AA, Kahn RS (2008) Microglia activation in recent-onset schizophrenia: a quantitative (R)-[11C] PK11195 positron emission tomography study. Biol Psychiatry 64:820-822

15. Ikarashi Y, Mizoguchi K (2016) Neuropharmacological efficacy of the traditional Japanese Kampo medicine yokukansan and its active ingredients. Pharmacol Ther 166:84-95

16. Liu Y, Nakamura T, Toyoshima T, Lu F, Sumitani K, Shinomiya A, Keep RF, Yamamoto T, Tamiya T, Itano T (2014) Ameliorative effects of yokukansan on behavioral deficits in a gerbil model of global cerebral ischemia. Brain Res 16:300-307

17. Furuya M, Miyaoka T, Tsumori T, Liaury K, Hashioka S, Wake R, Tsuchie K, Fukushima M, Ezoe S, Horiguchi J (2013) Yokukansan promotes hippocampal neurogenesis associated with the suppression of activated microglia in Gunn rat. J Neuroinflammation 10:145

18. Gudi V, Gingele S, Skripuletz T, Stangel M, Hermann D, Weissert R (2014) Glial response during cuprizone-induced de-and remyelination in the CNS: lessons learned. Front Cell Neurosci $8: 1-24$

19. Matsushima GK, Morell P (2001) The neurotoxicant, cuprizone, as a model to study demyelination and remyelination in the central nervous system. Brain Pathol 11:107-116

20. McMahon EJ, Cook DN, Suzuki KM (2001) Absence of macrophage-inflammatory protein-1alpha delays central nervous system demyelination in the presence of an intact blood-brain barrier. J Immunol 167:2964-2971

21. Bakker DA, Ludwin SK (1987) Blood-brain barrier permeability during cuprizone-induced demyelination: implication for the patho-genesis of immune-mediated demyelinating diseases. J Neurol Sci 78:125-137

22. Mason JL, Suzuki K, Chaplin DD, Matsushima GK (2001) Interleukin-1beta promotes repair of the CNS. J Neurosci 21:7046-7052

23. Arnett HA, Hellendall RP, Matsushima GK, Suzuki K, Laubach VE, Sherman P, Ting JP (2002) The protective role of nitric oxide in a neurotoxicant-induced demyelinating model. J Immunol $168: 427-433$

24. Makinodan M, Yamauchi T, Tatsumi K, Okuda H, Noriyama Y, Sadamatsu M, Kishimoto T, Wanaka A (2009) Yi-Gan San restores behavioral alterations and a decrease of brain glutathione level in a mouse model of schizophrenia. J Brain Dis 1:1-6

25. Tanaka T, Murakami K, Bando Y, Yoshida S (2015) Interferon regulatory factor 7 participates in the M1-like microglial polarization switch. Glia 63:595-610

26. Nomura T, Bando Y, Bochimoto H, Koga D, Watanabe T, Yoshida S (2013) Three-dimensional ultra-structures of myelin and the axons in the spinal cord: application of SEM with the osmium maceration method to the central nervous system in two mouse models. Neurosci Res 75:190-197

27. Bando Y, Nomura T, Bochimoto H, Murakami K, Tanaka T, Watanabe T, Yoshida S (2015) Abnormal morphology of myelin and axon pathology in murine models of multiple sclerosis. Neurochem Int 81:16-27

28. Blasi E, Barluzzi R, Bocchini V, Mazzolla R, Bistoni F (1990) Immortalization of murine microglial cells by a v-raf/v-myc carrying retrovirus. J Neuroimmunol 27:229-237

29. Nakatani Y, Tsuji M, Amano T, Miyagawa K, Miyagishi H, Saito A, Imai T, Takeda K, Ishii D, Takeda H (2014) Neuroprotective effect of yokukansan against cytotoxicity induced by corticosterone on mouse hippocampal neurons. Phytomedicine 21:1458-1465

30. Kawakami Z, Kanno H, Ueki T, Terawaki K, Tabuchi M, Ikarashi Y, Kase Y (2009) Neuroprotective effects of yokukansan, a 
traditional Japanese medicine, on glutamate-mediated excitotoxicity in cultured cells. Neuroscience 159:1397-1407

31. Hiremath M, Saito Y, Knapp G, Ting J, Suzuki K, Matsushima G (1998) Microglial/macrophage accumulation during cuprizone-induced demyelination in C57BL/6 mice. J Neuroimmunol 92:38-49

32. Clarner T, Janssen K, Nellessen L, Stangel M, Skripuletz T, Krauspe B, Hess FM, Denecke B, Beutner C, Linnartz-Gerlach B, Neumann H, Vallières L, Amor S, Ohl K, Tenbrock K, Beyer C, Kipp M (2015) CXCL10 triggers early microglial activation in the cuprizone model. J Immunol 194:3400-3413

33. Pasquini LA, Calatayud CA, Bertone Uña AL, Millet V, Pasquini JM, Soto EF (2007) The neurotoxic effect of cuprizone on oligodendrocytes depends on the presence of pro-inflammatory cytokines secreted by microglia. Neurochem Res 32:279-292

34. Morita S, Tatsumi K, Makinodan M, Okuda H, Kishimoto T, Wanaka A (2014) Geissoschizine methyl ether, an alkaloid from the uncaria hook, improves remyelination after cuprizone-induced demyelination in medial prefrontal cortex of adult mice. Neurochem Res 39:59-67

35. Masson JL, Jones JJ, Taniike M, Morell O, Suzuki K, Matsushima GK (2000) Mature oligodendrocyte apoptosis precedes IGF-1 production and oligodendrocyte progenitor accumulation and differentiation during demyelination/remyelination. J Neurosci Res 61:251-262

36. Stansley B, Post J, Hensley K (2012) A comparative review of cell culture systems for the study of microglial biology in Alzheimer's disease. J Neuroinflammation 9:115

37. Henn A, Lund S, Hedtjärn M, Schrattenholz A, Pörzgen P, Leist M (2009) The suitability of BV2 cells as alternative model system for primary microglia cultures or for animal experiments examining brain inflammation. ALTEX 26:83-94

38. Yrjänheikki J, Keinänen R, Pellikka M, Hökfelt T, Koistinaho J (1998) Tetracyclines inhibit microglial activation and are neuroprotective in global brain ischemia. Proc Natl Acad Sci USA 95:15769-15774

39. Skripuletz T, Miller E, Moharregh-Khiabani D, Blank A, Pul R, Gudi V, Trebst C, Stangel M (2010) Beneficial effects of minocycline on cuprizone induced cortical demyelination. Neurochem Res 35:1422-1433

40. Giulian D, Lachman LB (1985) Interleukin-1 stimulation of astroglial proliferation after brain injury. Science 228:497-499

41. Giulian D, Woodward J, Young DG, Krebs JF, Lachman LB (1988) Interleukin-1 injected into mammalian brain stimulates astrogliosis and neovascularization. J Neurosci 8:2485-2490

42. Giulian D, Baker TJ, Shih LC, Lachman LB (1986) Interleukin 1 of the central nervous system is produced by ameboid microglia. J Exp Med 164:594-604

43. Imamura S, Tabuchi M, Kushida H, Nishi A, Kanno H, Yamaguchi T, Sekiguchi K, Ikarashi Y, Kase Y (2011) The blood-brain barrier permeability of geissoschizine methyl ether in Uncaria hook, a galenical constituent of the traditional Japanese medicine yokukansan. Cell Mol Neurobiol 31:787-793

44. Mizoguchi K, Kushida H, Kanno H, Igarashi Y, Nishimura H, Ikarashi Y, Kase Y (2014) Specific binding and characteristics of geissoschizine methyl ether, an indole alkaloid of Uncaria Hook, in the rat brain. J Ethnopharmacol 158:264-270

45. Han IO, Kim KW, Ryu JH, Kim WK (2002) p38 mitogen-activated protein kinase mediates lipopolysaccharide, not interferongamma, -induced inducible nitric oxide synthase expression in mouse BV2 microglial cells. Neurosci Lett 325:9-12

46. Lampron A, Larochelle A, Laflamme N, Préfontaine P, Plante MM, Sánchez MG, Yong VW, Stys PK, Tremblay MÈ, Rivest S (2015) Inefficient clearance of myelin debris by microglia impairs remyelinating processes. J Exp Med 212:481-495

47. Neumann H, Kotter MR, Franklin RJ (2009) Debris clearance by microglia: an essential link between degeneration and regeneration. Brain 132:288-295 